\title{
Theoretical study of Aniline-Thiophene based Polymers
}

\author{
P. P. Zamora ${ }^{1}$, M. B. Camarada², F. R. Díaz ${ }^{3}$, M. A. del Valle ${ }^{4}$, L. Cattin ${ }^{5}$, G.Louarn ${ }^{5}$, M. \\ Soler ${ }^{1}$ and J.C. Bernède ${ }^{6}$
}

1 Universidad de Chile, Facultad de Ciencias Físicas y Matemáticas, Departamento de Ciencia de Materiales, Av. Tuper 2069, Santiago, Chile

2 Universidad Andres Bello, Facultad de Biología, Center for Bioinformatics and Integrative Biology (CBIB), Republica 239, Santiago, Chile

3 Pontificia Universidad Católica de Chile, Facultad de Química, Departamento de Química Orgánica, Laboratorio Polímeros conductores, Av. Vicuña Mackenna 4860, Santiago de Chile

4 Pontificia Universidad Católica de Chile, Facultad de Química, Departamento de Química Inorgánica, Laboratorio de Electroquímica, Av. Vicuña Mackenna 4860, Santiago de Chile

5 Université de Nantes, Institut des Matériaux Jean Rouxel (IMN), CNRS, UMR 6205, 2 rue de la Houssinière, BP 32229, 44322 Nantes cedex 3, France.

6 Université de Nantes, MOLTECH-Anjou, CNRS, UMR 6200, 2 rue de la Houssinière,

\section{ABSTRACT}

In this work, two kinds of novel thiophene-aniline polymers, were studied through theoretical protocols in order to find new insights about the polymerization mechanism, the chemical structure and optical properties exhibited by this kind of conducting materials. The dual descriptor - a local reactivity descriptor derived from conceptual density functional theoryand the condensed electrophilic Fukui index were used to describe the most probable sites of polymerization. Frontier orbitals energies were calculated to predict the band gap of both polymeric films. All results demonstrated how theoretical protocols help in the understanding of chemical polymerization mechanism and in the prediction of relevant properties, which can accelerate the synthesis process of new monomers.

\section{Indexing terms/Keywords}

Conducting polymers; DFT; band gap; dual reactivity index.

\section{Academic Discipline And Sub-Disciplines}

Theoretical chemistry, Solar energy

\section{SUBJECT CLASSIFICATION}

Paper

\section{TYPE (METHOD/APPROACH)}

Theoretical study of monomers with photovoltaics properties

\section{Council for Innovative Research}

Peer Review Research Publishing System

\section{Journal: Journal of Advances in Chemistry}

Vol. 6, No. 2

editor@cirworld.com

www.cirworld.com, member.cirworld.com 


\section{INTRODUCTION}

Conducting polymers are conjugated organic materials well known for their exceptional environmental stability, processability and electroactivity. In recent years they have found worldwide attention because of their application in a diversity of devices such as organic light emitting diodes ${ }^{1,2}$, photovoltaics ${ }^{2}$, supercapacitors ${ }^{3}$ and biosensors ${ }^{4}$. This kind of materials can be synthesized by electrochemical and chemical means, the last corresponding to the traditional organic route, where an oxidizing agent is added to activate the monomers and produce cationic radicals, which react and generate the conducting film.

The ultimate utility of conducting polymers relies on the ability to optimize properties for a given application, for example to improve conductivity, the energy difference between frontier orbitals can be tuned by the synthesis of appropriate monomers capable of reducing this quantity. This is the case of two novel monomers and their corresponding polymers: 4(2-thiophen)-aniline (4,2TA) and 4-(3-thiophen)-aniline (4,3TA) (see Figure 1) which are electron donor, and exhibit a reduced band gap $\left(E_{g}\right)^{5-7}$. These two monomers and their corresponding polymers, poly 4-(2-thiophen)-aniline (P4,2TA) and poly 4-(3-thiophen)-aniline (P4,3TA), were already characterized by experimental techniques ${ }^{5}$, however no electronic distribution evidence was found to clearly explain the polymerization mechanism. In this sense computational chemistry is a valuable tool to complement experimental studies. It delivers new insights on material science, helps in the rational designing process of new molecular structures with improved properties and gives relevant information about the density and electronic structure.

On the other hand, to accelerate the efforts in technology development and succeed in the designing of new and improved materials, it is significant to find the relationship between photoelectrical properties and electronic structure. In this sense, theoretical protocols could be very useful to calculate and predict intrinsic properties of conducting polymers, like the energy gap between frontier orbitals $\left(E_{g}\right)$ HOMO and LUMO.

In this work, we performed theoretical calculations to find new insights about the polymerization mechanism of 4,2TA and 4,3TA such as the most probable sites of polymerization, and in this way understand the reported structure of the conducting film in our previous work ${ }^{5}$. HOMO and LUMO energies were used to predict the band gap of both polymeric films. These results demonstrated how theoretical protocols could help in the understanding of chemical polymerization mechanism and in the prediction of relevant properties which can accelerate the synthesis process of new monomers.

\section{COMPUTATIONAL BACKGROUND}

The HOMO energy $\left(\mathrm{E}_{\mathrm{HOMO}}\right)$ corresponds to the necessary energy to remove an electron from a molecule. The Koopmans

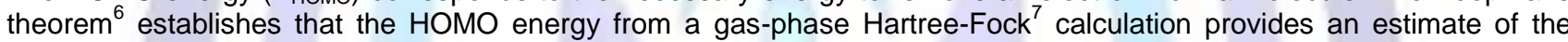
vertical ionization potential, ignoring relaxation effects of the ionization process and the electronic correlation. While Koopmans theorem does not apply to DFT HOMO eigen-values, they can still often be correlated with ionization potentials (IP). Then, IP = - $E_{\text {HOMO }}$. Within the same framework, LUMO energy ( $E_{\text {LUMO }}$ ) can be approximated to the electron affinity (EA). The energy band gap $(\mathrm{Eg})$ corresponds to the energy difference between the respective eigenvalues of the frontier molecular orbitals, as Eq. 1 shows:

$$
\mathrm{Eg}=\left(\mathrm{E}_{\mathrm{LUMO}}-\mathrm{E}_{\mathrm{HOMO}}\right) \mathrm{eV}
$$

The understanding of the polymerization mechanisms is also a relevant aspect. This issue can be investigated through local electronic descriptors defined in the so-called Conceptual Density Functional Theory ${ }^{8,9}$, such as the Fukui function $f(\vec{r})^{10}$, and the dual descriptor of chemical reactivity $\Delta f(\vec{r})$. The Fukui function was first defined by Parr and Yang ${ }^{11}$ as the derivative of the chemical potential $(\mu)$ with respect to the external potential $\left({ }^{u}(\vec{r})\right.$ ) or equivalently as the derivative of the total electron density $\left({ }^{\mathscr{P}} \vec{p}\right)$ with respect to the total number of electrons, $N$ :

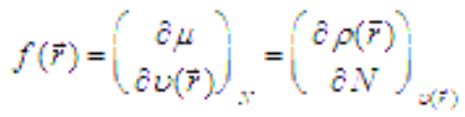

The Fukui function reflects the character of a molecule to accept (donate) electrons from (to) another system. For a molecular system, the density is a discrete function of the number of electrons. Because of the discontinuity of the derivative on the right-hand of the Eq. (2) at an integer value of $\mathrm{N}$, three different physical meanings can be associated to the left, right, and central derivative of the density with respect to $\mathrm{N}$, corresponding to reactivity indexes for a nucleophilic, $f^{*}(\vec{P})$ electrophilic $f^{-(\vec{y})}$, and radical $f^{0}(\vec{P})$ attacks, respectively.

The nucleophilic and electrophilic Fukui functions can be approximated as the density of the frontier molecular orbitals as $f^{a}(\vec{r}) \approx \rho_{\text {conso }}(\vec{r})$ and $\hat{f}^{-}(\vec{r}) \approx \rho_{\text {sons }}(\vec{r})$, respectively. By applying the finite difference approximation and population analysis, condensed Fukui indexes for each atom in a molecule can be expressed as follows: 


$$
\begin{aligned}
f_{8}^{*} & \approx\left[N_{g}(N+1)-N_{g}(N)\right] \\
f_{8} & \approx\left[N_{g}(N)-N_{g}(N-1)\right]
\end{aligned}
$$

where $N_{k}$ corresponds to the electronic population on the atom $k$ for the systems with $(\mathrm{N}-1)$, $(\mathrm{N})$, and $(\mathrm{N}+1)$ electrons, evaluated at the same geometry with $\mathrm{N}$ electrons.

The dual descriptor of chemical reactivity $(\Delta f(\vec{\gamma}))$ identifies simultaneously the nucleophilic and electrophilic regions within a molecule ${ }^{12,13}$. It is defined in terms of the variation of $f(\bar{r})$ with respect to the total number of electrons. Applying the finite difference approximation $\Delta f(\vec{\gamma})$ can be written as the difference between nucleophilic and electrophilic Fukui functions:

$$
\Delta f(\vec{y})=f^{t}(\vec{y})-f^{-}(\vec{y})
$$

If $\Delta f(\vec{r})>0$, the site is favored for an electrophilic attack, whereas if $\Delta f(\vec{r})<0$ it is favored for a nucleophilic attack.

\section{COMPUTATIONAL METHODOLOGY}

All calculations were based on the Density Functional Theory ${ }^{14,15}$ using the computational package Gaussian03 ${ }^{16}$. Geometry optimizations were done using the hybrid exchange-correlation B3LYP ${ }^{17-19}$ functional, combined with the triple split-valence basis set and polarization functions $6-311 \mathrm{G}(\mathrm{d}, \mathrm{p})$. The selection of this level of theory was based on a previous study done by Duarte et al. ${ }^{20}$ of the potential energy surface of $2 \mathrm{Th}$, where several density functionals as well as CCSD (coupled-cluster with single and double excitations) and MP4(SDQ) (fourth order Møller-Plesset perturbation theory, neglecting triple excitations) where selected. Regarding the structural predictions they found that the B3LYP/6$311 \mathrm{G}(\mathrm{d}, \mathrm{p})$ and MP2/cc-pVDZ fully optimized geometries exhibit the best agreement with the gas phase electron diffraction data, and concluded that DFT/6-311G** calculations were the most computationally efficient procedure.

Frequency calculations of all optimized structures were performed to confirm the nature of the stationary points. An unrestricted calculation scheme was applied for the oxidized species, i.e. the polarons, $4,2 \mathrm{TA}^{+}$and $4,3 \mathrm{TA}^{+}$, using single point calculations on neutral optimized geometries.

\section{RESULTS AND DISCUSSION}

\section{Molecular Structure of 4,2TA, 4,3TA, P4,2TA and P4,3TA}

In order to identify stable geometries for neutral monomers in gas phase, a conformational analysis of 4,2TA and 4,3TA was done by changing the inter-ring torsional angle $(\alpha)$, defined by the $C_{T h}-C_{T h}-C_{A n}-C_{A n}$ backbone, every 10 degrees. Geometries were computed at B3LYP/6-311G(d,p) level and were fully relaxed at each point. In both cases, an antigauche conformer, at approx. $\alpha=150^{\circ}$, was found as the most stable structure in gas phase. In accordance to the experimental information, $4,2 \mathrm{TA}$ is $0.8 \mathrm{kcal} \cdot \mathrm{mol}^{-1}$ more stable than 4,3TA.

With the aim of determining the geometrical effects of aniline on thiophene, thiophene dimer structural parameters ${ }^{21}$ were compared to the optimized copolymers geometries. All thiophene ring bond lengths and angles of 4,2TA and 4,3TA exhibited less than $0.3 \%$ of difference in comparison to the thiophene dimer. In both cases, the length of the inter-ring bond was longer than the one of the thiophene dimer in $1.70 \%$ and $1.05 \%$ for $4,3 \mathrm{TA}$ and $4,2 \mathrm{TA}$, respectively. All structures presented the same inter-ring dihedral angle ${ }^{21-24}$, indicating that there are no important structural modifications of a thiophene dimer, when one thiophene ring is replaced by aniline.

Considering the optimized monomers as starting units, optimizations and frequency calculations of oligomers from 1 to 7 units were done. The heptamer was taken as an approximation to describe each polymeric systems, P4,2TA and P4,3TA, based on the emeraldine-type structure of the P4,2TA.

\section{Condensed electrophilic Fukui index and dual descriptor}

The local properties associated to the oxidation processes, such as the most probable sites of polymerization, are very important to understand how the electron density is reorganized. These aspects have been explored through the condensed electrophilic Fukui index $\hat{f}_{8}$ and the reactivity dual descriptor $\Delta f(\vec{y})$.

According to experimental ${ }^{1} \mathrm{H}-\mathrm{NMR}$ evidence ${ }^{5}$, the polymerization reaction of 4,2TA takes place between the nitrogen atom of aniline and the C5 of the thiophene ring, as well as between the C5 of two thiophene rings. Consequently, there are only two possible polymerization positions giving rise to a linear polymeric structure, as Figure $2 a$ depicts. Since the nitrogen-nitrogen linkage is not possible in this type of polymerization processes ${ }^{25}$, only one C5-C5 bond per polymeric chain can exist to warrantee the polymer chain development.

In the case of 4,3TA, the polymerization occurs through $\mathrm{C} 2$ and $\mathrm{C} 4$ of the thiophene ring. No evidence of $\mathrm{C}_{T h}-\mathrm{C}_{T h}$ linkage (C2-C2, C4-C4 or C4-C2). Therefore, P4,3TA presents two possible sites of polymerization, which leads to the formation of a non-linear, and branched structure, without quinoidal units (Figure $2 b$ ). 
(a)

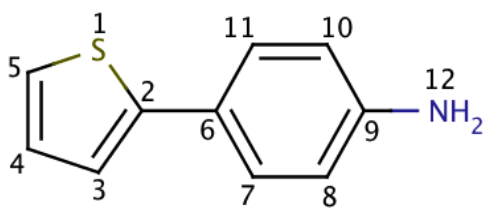

(b)

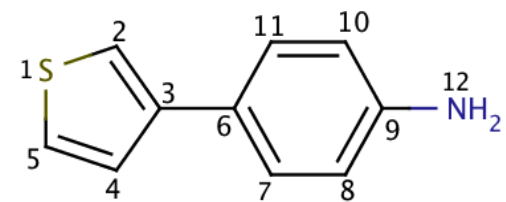

Figure 1. Chemical structure of the synthesized monomers, (a) 4-(2-thiophen)-aniline (4,2TA) and (b) 4-(3thiophen)-aniline $(4,3 T A)$

The condensed electrophilic Fukui index was calculated using the neutral optimized monomers and polarons, 4,2TA ${ }^{+.}$and 4,3 $\mathrm{TA}^{+}$. Table 1 (see Figure 1 for atomic numeration) summarizes $f_{8}^{-}$values of 4,2TA and 4,3TA. In both monomers, the $\mathrm{N}$ atom is the place with the highest value, i.e. the place with more electronic density, susceptible to electrophilic attacks. Then, when the oxidizing agent is added, this is the most probable position for the polaron formation. In the case of 4,2TA, the second most nucleophilic atom is the carbon number five. Therefore, N and C5 are the most probable places of polymerization generating a lineal polymeric chain, which is in accordance with the NMR spectroscopy ${ }^{5}$ where the polymer exhibited bonds at the $\mathrm{C} 5$ and $\mathrm{N}$ atom. On the other hand, when the $f_{8}$ values on the thiophene ring of 4,3TA are analyzed, C2 presents the highest nucleophilic character, which coincides with the spectroscopic and electrochemical analysis already reported ${ }^{5}$, where the polymer exhibited a branched structure because of the union of C2. However, contrary to the experimental evidence, C5 has a $f($ value higher than C4. Probably, during the organic synthesis the radical coupling via C5 also occurs, but in less quantity in comparison to the C4 reaction path, being not enough for the ${ }^{1} \mathrm{H}-\mathrm{NMR}$ detection ${ }^{5}$.

\begin{tabular}{l|cc|c|cc}
\hline \hline Atom & 4,2TA & 4,3TA & \multicolumn{1}{l}{ Atom } & \multicolumn{4}{c}{ 4,2TA } & 4,3TA \\
\hline S1 & 0.068 & 0.096 & C7 & 0.031 & 0.099 \\
C2 & 0.027 & 0.300 & C8 & 0.056 & 0.191 \\
C3 & 0.080 & 0.084 & C9 & 0.053 & 0.010 \\
C4 & 0.028 & 0.224 & C10 & 0.054 & 0.188 \\
C5 & 0.123 & 0.233 & C11 & 0.019 & 0.098 \\
C6 & 0.090 & 0.192 & N12 & 0.127 & 0.573 \\
\hline \hline
\end{tabular}

Table 1. Electrophilic Fukui function $f^{-}(\vec{P})$ values calculated at B3LYP/6-311g(d,p) level. For atomic numeration see Figure 1.

In 4,2TA, the condensed electrophilic Fukui index of the $\mathrm{N}$ and $\mathrm{C} 5$ are almost the same, both atomic positions have the same probability of being radical sites. Therefore, a C5 attack to a C5`s neutral molecule could be possible, as the experimental evidence showed. On the other hand, the nitrogen atom at 4,3TA, exhibited a $f_{\&}$ value almost 2.5 times higher than C4 and C5, and approx. two times upper than the value on C2. This indicates that there is a low probability of having activated carbon positions at the thiophene ring. Then, no inter-ring $\mathrm{C}_{\mathrm{Th}}-\mathrm{C}_{\mathrm{Th}}$ bonds will be created in accordance with the experimental results above mentioned (Figure $2 b$ ).

For the analysis of the dual descriptor of chemical reactivity ${ }^{12,13}$, the neutral optimized forms of 4,2 TA and 4,3TA were used. The maps of the dual descriptor of each monomer are displayed in Figure 3 . It can be observed that the nitrogen atom region is one of the nucleophilic zones present in both molecules. As the $f_{\&}$ analysis pointed out, there is a high charge concentration on this atom, and once the oxidizing agent is added, the polaron formation is more probable on this site. In the case of 4,2TA the $f_{8}$ index on $\mathrm{C} 5$ was very close to the $\mathrm{N}$ value, and then, $\mathrm{N}$ or $\mathrm{C} 5$ radical attacks are possible, pointed towards a nucleophilic site of another 4,2TA neutral molecule. Analyzing the $\Delta f(\vec{r})$ map (Figure $3 a)$, C5 presents a totally nucleophilic character, contrary to $\mathrm{C} 3$ and $\mathrm{C} 4$. Therefore, $\mathrm{C} 5-\mathrm{N}$ or $\mathrm{C} 5-\mathrm{C} 5$ linkages will be present in $\mathrm{P} 4,2 \mathrm{TA}$, being in accordance with the lineal polymeric chain observed in the ${ }^{1} \mathrm{H}-\mathrm{NMR}$ spectroscopy ${ }^{5}$. 
(a)

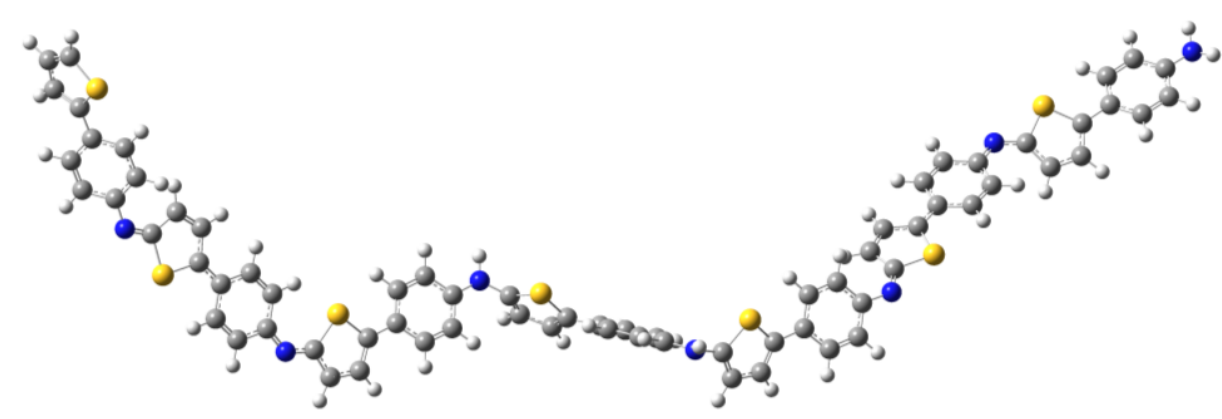

(b)

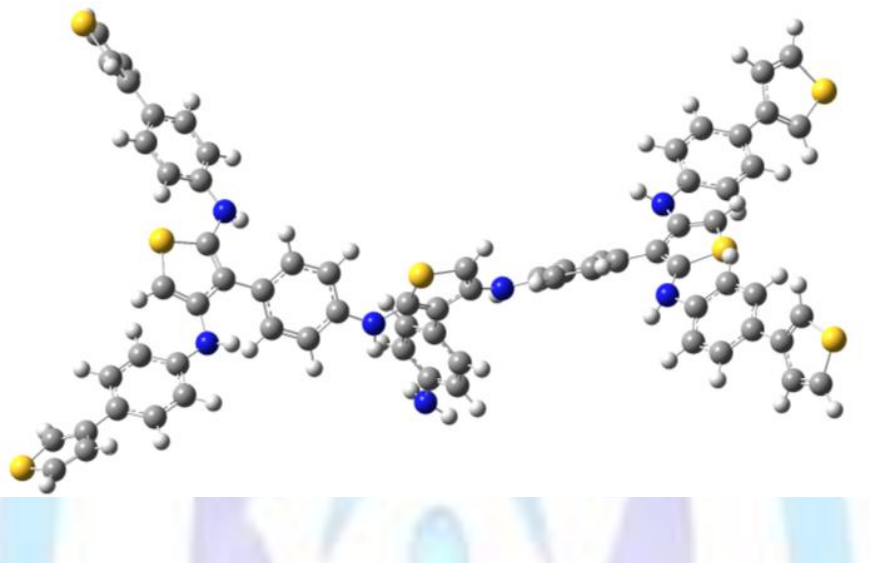

Figure 2. Optimized heptamer structure of the (a) quinoidal 4,2TA and (b) 4,3TA at B3LYP/6-311g(d,p)

Figure $3 \mathrm{~b}$ depicts the nucleophilic zones at the thiophene ring of 4,3TA: C2, C4 and C5. Experimentally only the polymerization through $\mathrm{N}-\mathrm{C} 2$ and $\mathrm{N}-\mathrm{C} 4$ was observed. As already mentioned, probably the radical coupling via $\mathrm{N}-\mathrm{C} 5$ also occurs in minor amounts, but no structures were detected through the experimental analysis.

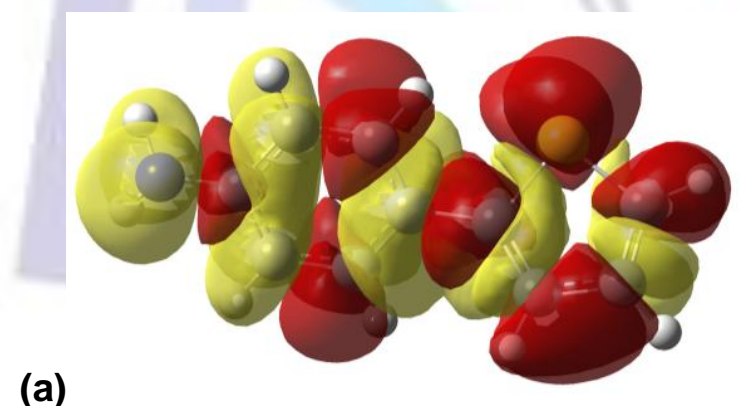

(a)

(b)

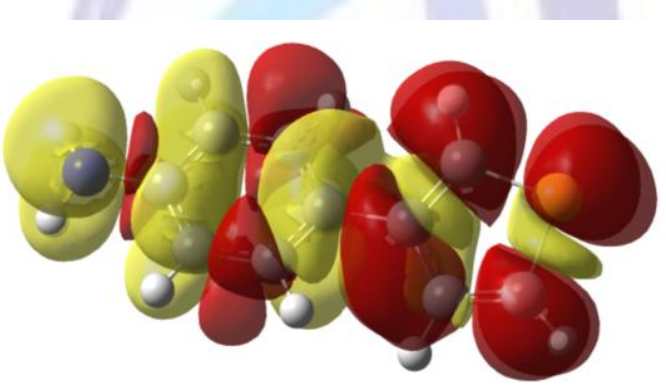

Figure 3. ${ }^{\Delta f(\vec{v})}$ map for the neutral (a) 4,2TA and (b) 4,3TA monomers. Electrophilic regions $(\stackrel{\Delta f(\vec{r})}{>}>0$ ) are indicated in yellow and the nucleophilic regions $(\Delta f(\vec{r})<0)$ are in red

\section{Prediction of the band gap of P4,2TA and P4,3TA}

The Eg, HOMO and LUMO values of P4,2TA and P4,3TA were calculated using an heptamer as model for the polymer. In the case of 4,2TA no quinoidal units were considered, and as already mentioned, only one C5-C5 bond per polymeric chain can exist. Therefore no $\mathrm{C}_{5}-\mathrm{C}_{5}$ linkages of thiophene ring were considered in the model because there is only one of these unions per polymeric chain. Figure 2 shows both optimized structures.

The theoretical band gap (Eg) energy for each 4,2TA and 4,3TA oligomer (from 1 to 7 units) was calculated as the energy difference between the respective eigenvalues of the frontier molecular orbitals using the Eq. 2. Table 2. collects all Eg's. 
The band gap can be extrapolated for very long backbones, by using the Meier expression ${ }^{26}$ (Eq. 5), which had successfully applied to describe the absorption and fluorescence values of polymers as the oligomer chain grows:

$$
E g(x)=E g_{m}+\left(E g_{1}-E g_{g}\right) e^{-s(g-1)}
$$

$\mathrm{Eg}_{1}$ is the $\mathrm{Eg}$ for the monomer unit, $\mathrm{Eg}_{\infty}$ is the band gap value at infinite chain length, i.e. P4,2TA or P4,3TA, a corresponds to the fitting parameter and $\mathrm{n}$ is the number of repeating units in the oligomer structures.

\begin{tabular}{c||ccc||ccc||ccc}
\hline \hline \multicolumn{1}{c||}{} & \multicolumn{3}{c||}{ aromatic 4,2TA } & \multicolumn{3}{c||}{ quinoidal 4,2TA } & \multicolumn{3}{c}{ 4,3TA } \\
\hline Units & - & - -LUMO & Eg & - & -LUMO & Eg & - & -LUMO & Eg \\
& HOMO & & & HOMO & & & & \\
\hline 1 & 5.332 & 0.835 & 4.497 & 5.332 & 0.835 & 4.497 & 5.390 & 0.625 & 4.765 \\
2 & 5.291 & 1.041 & 4.250 & 5.196 & 1.094 & 4.250 & 5.066 & 0.820 & 4.246 \\
3 & 5.272 & 1.137 & 4.135 & 4.877 & 3.280 & 1.597 & 4.963 & 0.975 & 3.988 \\
4 & 5.222 & 1.174 & 4.048 & 4.884 & 3.304 & 1.580 & 4.962 & 1.041 & 3.921 \\
5 & 5.220 & 1.204 & 4.016 & 4.924 & 3.335 & 1.589 & 4.958 & 1.101 & 3.857 \\
6 & 5.215 & 1.218 & 3.997 & 4.937 & 3.346 & 1.592 & 4.960 & 1.187 & 3.773 \\
7 & 5.198 & 1.205 & 3.993 & 4.826 & 3.364 & 1.462 & 4.944 & 1.231 & 3.712 \\
polyme & 5.174 & 1.218 & 3.976 & - & - & - & 4.951 & 1.324 & 3.740 \\
r & & & & & & & & & \\
\hline
\end{tabular}

Table 2. Predicted (B3LYP/6-311G**) values of HOMO, LUMO and the optical bang gap (Eg) of the aromatic and quinoidal 4,2TA and 4,3TA oligomers. All values in $\mathrm{eV}$.

Figure 4 depicts the Eg data of the aromatic 4,2TA and 4,3TA oligomers, and the fitted curve adjusted with Eq. 5. In the case of $4,3 \mathrm{TA}$, the extrapolated band gap to the polymeric material is $3.74 \mathrm{eV}$, and $3.98 \mathrm{eV}$ for the aromatic $4,2 \mathrm{TA}$.

(a)

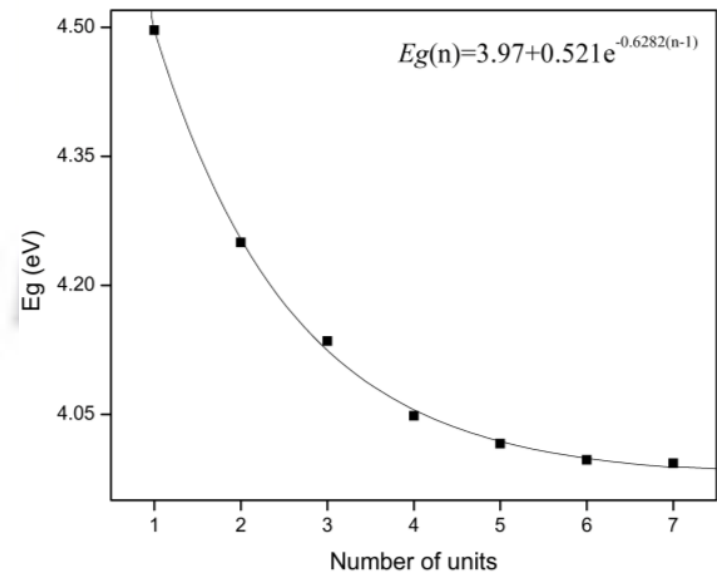

(b)

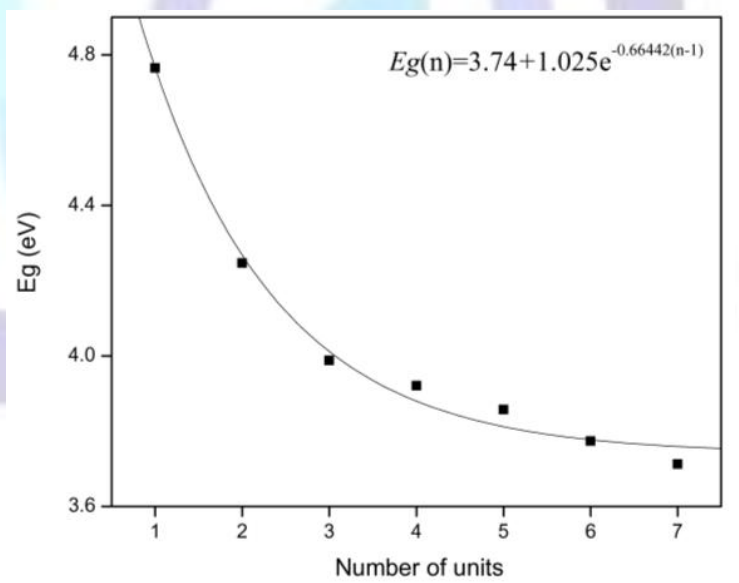

Figure 4. Band gap (eV) and the Meier fit across the calculated values of the (a) aromatic 4,2TA oligomers $\left(R^{2}\right.$ $=0.9990)$ and the (b) 4,3 TA oligomers $\left(R^{2}=0.9913\right)$

Both values are quite distant from the experimental UV-vis Eg value ${ }^{5}$ (Table 3), presenting a difference of 1.64 eV and $2.18 \mathrm{eV}$, respectively. For the energetic estimation of the frontier orbitals, in both cases the theoretical HOMO level is in accordance with the electrochemical HOMO value, however, the LUMO energy differs in 1.88 eV (aromatic 4,2TA) and $1.68 \mathrm{eV}$ (4,3TA). Then, the LUMO values are the main source of error in the Eg prediction. The Eg and LUMO overestimation indicates that the selected structures for the representation of the aromatic 4,2TA and the 4,3TA heptamer disagree from the real oligomeric geometry. In the case of 4,3TA, the incorporation of more cross-linked chains, C5 site polymerization and also the consideration of larger oligomers will improve the description of this quantity. 


\begin{tabular}{lccc}
\hline & -HOMO $(\mathrm{eV})$ & -LUMO $(\mathrm{eV})$ & Eg (eV) \\
\hline P4,2TA & 4.9 & 3.1 & 1.8 \\
P4,3TA & 5.1 & 3.0 & 2.1 \\
\hline \hline
\end{tabular}

*Values from Ref. 5

Table 3. Experimental values ${ }^{\star}$ of HOMO, LUMO and the optical bang gap (Eg) of P4,2TA and P4,3TA. All values in eV.

On the other hand, as the experimental evidence demonstrated ${ }^{5}$, the aromatic $4,2 \mathrm{TA}$ structure is less representative than the quinoidal form. New calculations were performed, considering this time the emeraldine oxidation state: one quinoidal unit per three benzenoic rings. Energetic data could not be simulated using the Meier equation, so the heptamer energetic values were approximated to the polymer. As can be seen in Table 2, the introduction of quinoidal rings from the trimer, immediately reduces the band gap as Alemán et al. previously reported ${ }^{27}$. The HOMO-LUMO energy levels are in accordance with the experimental values. The $\mathrm{Eg}$ is underestimated by approx. $0.3 \mathrm{eV}$. Theoretical calculations of the quinoidal structure coincide with the optical and electrochemical evidence ${ }^{5}$, demonstrating that the aromatic-type chain is not the appropriate representation of P4,2TA.

Considering the theoretical structures proposed for both polymeric systems, it can be inferred a better performance of P4,2TA when used in the fabrication of a solar cell. Its lineal conformation ${ }^{33-35}$ and lower band gap would enable a better contact with the buffer layer being more suitable than P4,3TA in photovoltaic devices. Therefore here the better performance of P4,2TA may be due to its lineal conformation because it allows to decrease the disorder in the film and to increase its conductivity, the fact that its band gap is smaller can help to increase the Jsc This evidence agrees well with the experimental results of the solar cell constructed in our previous work ${ }^{5}$, where P42TA showed a superior photovoltaic performance.

The predicted molecular structure of P4,2TA and P4,3TA, the optical values, and the reactivity index complement the experimental results, and contribute in the understanding and prediction of the optical and electrical properties of this kind of materials and possible new molecules with photovoltaic activity ${ }^{28-32}$.

\section{CONCLUSIONS}

Theoretical calculations agree very closely with the structural, optical and electronic experimental information. This evidence is reflected in the results of the Fukui index and frontier orbitals and demonstrates that theoretical studies can be an appropriate tool to understand and predict experimental molecular properties. In this sense, a good estimation of HOMO, LUMO and Eg, allows the prediction of central solar cell parameters such as the open circuit potential (Voc), conductivity (photocurrent) and possible orientations of polymer chains as a consequence of atomic interactions with the solar cell anode.

The dual reactivity index elucidated the polymerization positions and therefore, the polymerization mechanism of P4,2TA and P4,3TA. This information successfully explained the experimental evidence ( ${ }^{1} \mathrm{H}-\mathrm{NMR}$ spectroscopy).

\section{ACKNOWLEDGMENTS}

The authors thank FONDECYT financial support through project 24110046, granted to conduct this research and ECOS CONICYT project No.C09E02

\section{REFERENCES}

(1) Friend, R. H.; Gymer, R. W.; Holmes, A. B.; Burroughes, J. H.; Marks, R. N.; Taliani, C.; Bradley, D. D. C.; Santos, D. A. D.; Bredas, J. L.; Logdlund, M. Nature 1999, 397, 121.

(2) Brabec, C. J.; Sariciftci, N. S.; Hummelen, J. C. Advanced Functional Materials 2001, 11, 15.

(3) Hughes, M.; Chen, G. Z.; Shaffer, M. S. P.; Fray, D. J.; Windle, A. H. Chem. Mater. 2002, 14, 1610.

(4) Gerard, M.; Chaubey, A.; Malhotra, B. D. Biosensors and Bioelectronics 2002, 17, 345.

(5) Zamora, P. P.; Camarada, M. B.; Jessop, I. A.; Díaz, F. R.; del Valle, M. A.; Cattin, L.; Louarn, G.; Bernede, J. C. Int. J. Electrochem. Sci. 2012, 7, 8276.

(6) Koopmans, T. Physica 1934, 1, 104.

(7) Roothaan, C. C. J. Rev. Mod. Phys. 1951, 23, 69.

(8) Parr, R. G.; Yang, W. Density functional theory of atoms and molecules Oxford University Press: New York, 1997.

(9) Geerlings, P.; De Proft, F.; Langenaeker, W. Chem. Rev. 2003, 103, 1793.

(10) Fukui, K. Science 1987, 218, 747.

(11) Parr, R.; Yang, W. J. Am. Chem. Soc. 1984, 106, 4049. 
(12) Morell, C.; Grand, A.; Toro-Labbé, A. J. Phys. Chem. A 2005, 109, 205.

(13) Morell, C.; Grand, A.; Toro-Labbé, A. Chem. Phys. Lett. 2006, 425, 342.

(14) Hohenberg, P.; Kohn, W. Physical Review 1964, 136, B864.

(15) Kohn, W.; Sham, L. J. Phys. Rev. 1965, 140, A1133.

(16) Frisch, M. J.; Trucks, G. W.; Schlegel, H. B.; Scuseria, G. E.; Robb, M. A.; Cheeseman, J. R.; Montgomery, J. A.; Vreven, T.; Kudin, K. N.; Burant, J. C.; Millam, J. M.; Iyengar, S. S.; Tomasi, J.; Barone, V.; Mennucci, B.; Cossi, M.; Scalmani, G.; Rega, N.; Petersson, G. A.; Nakatsuji, H.; Hada, M.; Ehara, M.; Toyota, K.; Fukuda, R.; Hasegawa, J.; Ishida, M.; Nakajima, T.; Honda, Y.; Kitao, O.; Nakai, H.; Klene, M.; Li, X.; Knox, J. E.; Hratchian, H. P.; Cross, J. B.; Bakken, V.; Adamo, C.; Jaramillo, J.; Gomperts, R.; Stratmann, R. E.; Yazyev, O.; Austin, A. J.; Cammi, R.; Pomelli, C.; Ochterski, J. W.; Ayala, P. Y.; Morokuma, K.; Voth, G. A.; Salvador, P.; Dannenberg, J. J.; Zakrzewski, V. G.; Dapprich, S.; Daniels, A. D.; Strain, M. C.; Farkas, O.; Malick, D. K.; Rabuck, A. D.; Raghavachari, K.; Foresman, J. B.; Ortiz, J. V.; Cui, Q.; Baboul, A. G.; Clifford, S.; Cioslowski, J.; Stefanov, B. B.; Liu, G.; Liashenko, A.; Piskorz, P.; Komaromi, I.; Martin, R. L.; Fox, D. J.; Keith, T.; Laham, A.; Peng, C. Y.; Nanayakkara, A.; Challacombe, M.; Gill, P. M. W.; Johnson, B.; Chen, W.; Wong, M. W.; Gonzalez, C.; Pople, J. A. 2004.

(17) Becke, A. D. Phys. Rev. A 1988, 38, 3098.

(18) Lee, C.; Yang, W.; Parr, R. G. Phys. Rev. B 1988, 37, 785.

(19) Stephens, P. J.; Devlin, F. J.; Chabalowski, C. F.; Frisch, M. J. J. Phys. Chem. 1994, 98, 11623.

(20) Duarte, H. A.; Dos Santos, H. F.; Rocha, W. R.; De Almeida, W. B. Journal of Chemical Physics 2000, $113,4206$.

(21) Camarada, M. B.; Jaque, P.; Díaz, F. R.; del Valle, M. A. Journal of Polymer Science Part B: Polymer Physics 2011, 49, 1723.

(22) Raos, G.; Famulari, A.; Marcon, V. Chemical Physics Letters 2003, 379, 364.

(23) Karpfen, A.; Choi, C. H.; Kertesz, M. The Journal of Physical Chemistry A 1997, 101, 7426.

(24) Alemán, C.; Julia, L. The Journal of Physical Chemistry 1996, 100, 1524.

(25) Bhadra, S.; Singha, N.; Khastgir, D. Eur. Polym. J. 2008, 44, 1763.

(26) Meier, H.; Stalmach, U.; Kolshorn, H. Acta Polymerica 1997, 48, 379.

(27) Alemán, C.; Ferreira, C. A.; Torras, J.; Meneguzzi, A.; Canales, M.; Rodrigues, M. A.; Casanovas, J. Polymer 2008, 49, 5169.

(28) Krebs, F.C.; Sol. Energy Mater. Sol. Cells 2009, 93,394-412.

(29) Bernede, J.C.; Organic Photovoltaic cells: History, Principle and Techniques 2008,

53,1549-1564.

(30) Godoy, A.; Cattin, L.; Toumi, L; Diaz, F. R.; del Valle, M. A.; Soto, G. M.;

Kouskoussa, B.; Morsili, M.; Benchouk, K.; Khelil, A.; Bernede, J. C.; Sol. Energy

Mater. and Solar Cells 2010, 94,648-654.

(31) Bernede, J. C.; Cattin, L.; Morsli, M.; Berredjem, Y.; Energy Mater. Sol. Cells 2008, 92, 1508-1520.

(32) Shorotriya, V.; Li, Gi.; Yao, Y.; Chu, C-V.; Yang, Y. ; Appl. Phys. Lett 2006, 88,735-748.

(33) R. J. Kline a; M. D. McGehee Journal of Macromolecular Sciencew, Part C: Polymer Reviews, 46:27-45, 2006

(34) Lobana, T. S.; Khanna, S.; Castineiras, A.; Hundal, G.; Anorg, Z.; Allg. Chem 2010, 636, 454-456

(35) Gallego, A.; Castillo, O.; Zamora, F.; Delgado, S.; Inorg. Chem. 2002, 7, 1070- 1075 